\title{
Immunohistochemical Expression of Claudin-1 in Hashimoto's Thyroiditis and Graves' Disease Patients
}

\author{
Tatjana Zake1, Sandra Skuja1, Katrina Pekarska1, Valerija Groma ${ }^{1}$, Ilze Konrade², Regina Kleina ${ }^{3}$
}

${ }^{1}$ Institute of Anatomy and Anthropology, Riga Stradins University, Riga, Latvia,

2 Department of Internal Medicine, ${ }^{3}$ Department of Pathology, Riga Eastern Clinical University Hospital, Riga,

Latvia,

\section{Introduction}

Autoimmune thyroid diseases, including Graves' disease (GD) and Hashimoto's thyroiditis (HT), are complex diseases in which a combination of genetic susceptibility and environmental encounters leads to breakdown of immune tolerance.

Epidemiological and histological data indicate that thyroid cancer (TC) frequently occurs in the context of HT (Guarino et al. 2010), and that the association between HT and TC is antibody specific (Azizi et al. 2014). Several authors reported overexpression of claudin-1, protein of tight junctions, in invasive tumor cells, including TC, and it seems to be an early step in carcinogenesis for at least some cancer types.

\section{Objectives}

This study aimed to estimate expression of claudin- 1 in the thyroid gland tissue of patients with $\mathrm{HT}$ and GD assessed by immunohistochemistry.

\section{Methods}

35 paraffin-embedded thyroid gland tissue blocks from the adult patients undergoing thyroidectomy were obtained from Pathology Center of Riga East Clinical University Hospital. These 35 cases comprised 17 cases of HT, 7 of GD, and 11 cases of ordinary colloidal goiter with normal thyroid function served as controls. Immunohistochemical staining was performed using an anti-claudin-1 antibody. The expression levels were scored semiquantitatively.

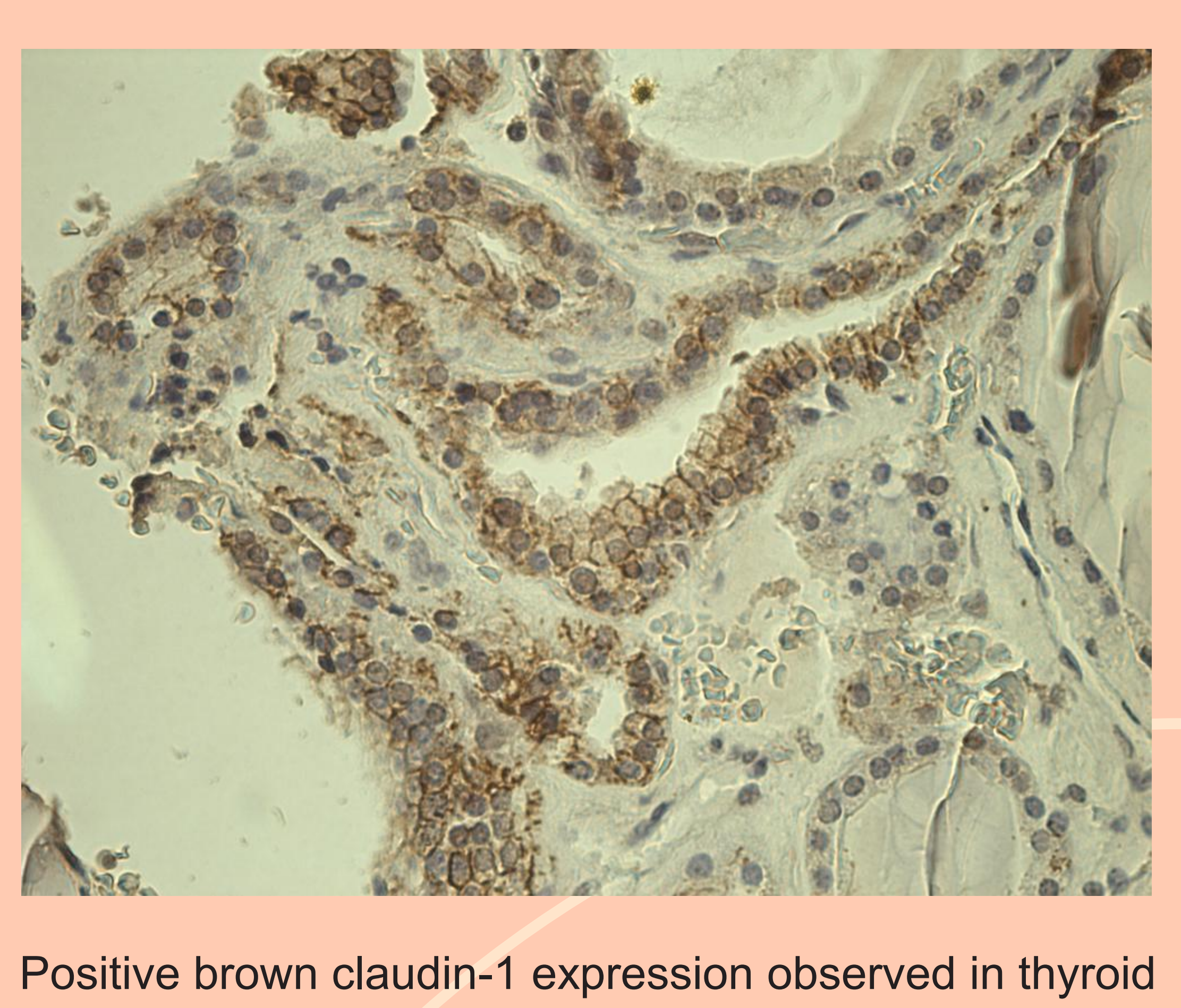
follicular cells in control group x400
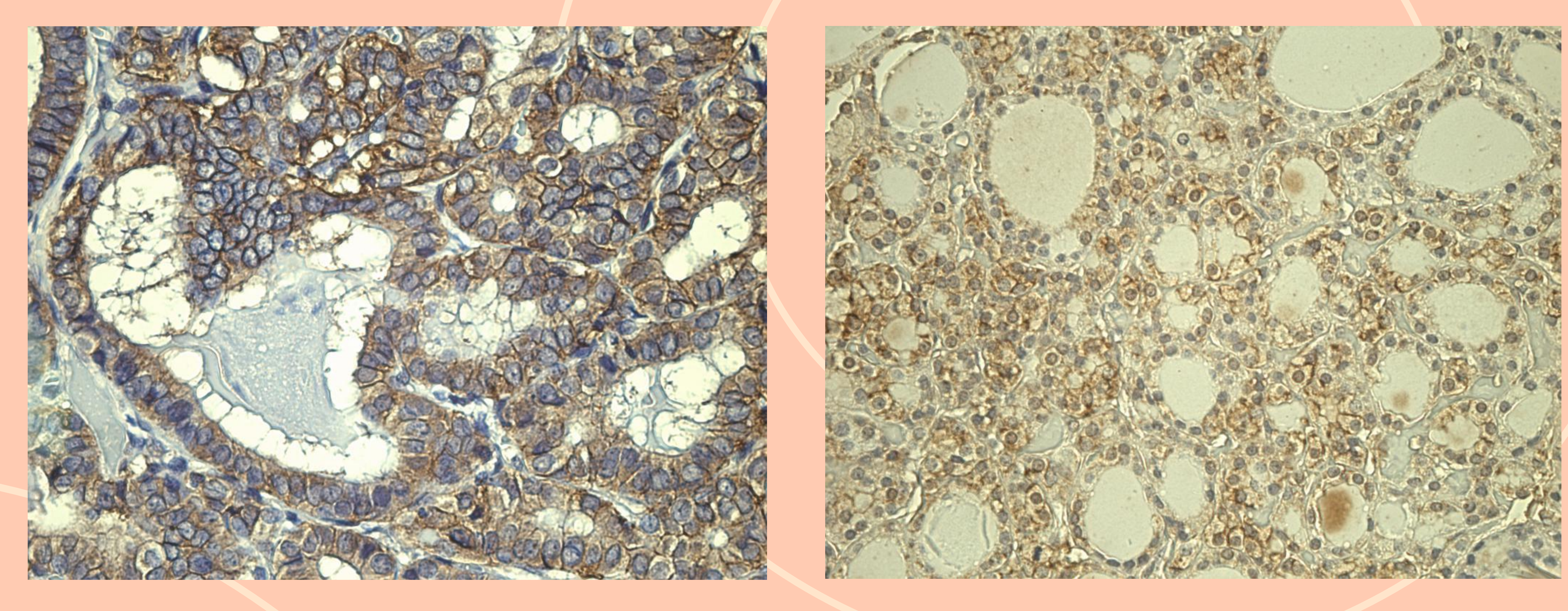

Diffuse circumferential membranous claudin-1 immunostaining in thyroid follicular cells of HT patients x400

\section{Results}

Claudin-1 showed a circumferential membranous staining pattern in thyroid follicular epithelial cells, positive claudin-1 expression (staining in $>5 \%$ of the cells) was observed in 27 out of 35 cases $(77.1 \%)$. The highest claudin- 1 mean expression level was observed in HT patients, although 6 out of 17 cases showed negative claudin-1 expression. The mean staining rate for claudin-1 in HT and GD thyroid follicular cells was calculated as $1.65 \pm 0.63$ and $1.22 \pm 0.19$, respectively, whereas in control group it was calculated as $1.58 \pm 0.44$. Only 8 out of 35 cases $(22.9 \%)$ showed claudin-1 positivity in $>50 \%$ of the cells (score 4 ); six of which were HT and 2 were colloidals goiters. The number of claudin-1 immunopositive thyroid follicles in HT patients was significantly higher than that in control group (Mann-Whitney test, $\mathrm{p}=0.030)$.

\section{Conclusions}

These results demonstrate that $\mathrm{HT}$ and GD patients display different junction protein expression patterns.

The overexpression of claudin-1 in HT when compared to control tissues and GD patients may be interpreted similarly to overexpression of claudins in epithelial-derived cancers, including TC, however, molecular mechanisms by which claudins affect tumorigenesis remain largely unknown.

\section{References:}

